\title{
Perfil dos Pacientes do Programa de Saúde Auditiva do Estado de Santa Catarina Atendidos no HU-UFSC
}

\section{Profile of Patients of the Auditory Health of the State of Santa Catarina Served at HU-UFSC}

\author{
Oscar Cardoso Dimatos *, Cláudio Márcio Yudi Ikino **, Paulo Arlindo Pbilippi***, \\ Spyros Cardoso Dimatos ****, Marília Susane Birck*, Paulo Fontoura Freitas *****.
}

* Médico (a) Graduado (a) pela Universidade Federal de Santa Catarina.

** Professor Adjunto da Universidade Federal de Santa Catarina. Doutor em Otorrinolaringologia pela Universidade de São Paulo.

*** Professor Adjunto da Universidade Federal de Santa Catarina.

**** Médico Otorrinolaringologista. Pós-graduando do Departamento de Otorrinolaringologia da Universidade Federal de São Paulo.

****** Médico Epidemiologista - Professor do Programa de Pós-graduação em Ciências Médicas CCS/HU/UFSC. Doutor em Epidemiologia pela Universidade de Londres - Reino Unido.

Instituição: Universidade Federal de Santa Catarina

Florianópolis / SC - Brasil.

Endereço para correspondência: Oscar Cardoso Dimatos - Avenida Jornalista Rubens de Arruda Ramos, 1010 , Apto 601 - Centro - Florianópolis / SC - Brasil - CEP 88015-700 - Telefone: (+55 48) 3225-2002 / (+55 48) 9914-2002 - E-mail: zicodimatos@yahoo.com.br

Artigo recebido em 21 de Outubro de 2010. Artigo aprovado em 13 de Novembro de 2010.

\section{RESUMO}

Introdução:

Objetivo:

Método:

Resultados:

Conclusão:

Palavras-chave:

\section{SUMMARY}

Introduction:

Objective:

Method:

Results:

Conclusion:

Keywords:
A audição é uma das funções essenciais para o desenvolvimento da linguagem e sua deficiência pode levar a diversas consequências.

Descrever o perfil dos pacientes do Programa de Saúde Auditiva do Estado de Santa Catarina atendidos no Hospital Universitário da Universidade Federal de Santa Catarina.

Realizou-se um estudo retrospectivo com os pacientes com indicação de protetização auditiva atendidos nos anos de 2007 e 2008. Dividiram-se os pacientes em 2 grupos: pediátrico ( $<18$ anos) e adulto ( $\geq 18$ anos). Avaliou-se: idade, sexo, tipo e grau de perda, duração, comorbidades e etiologia provável. Analisaram-se 304 prontuários, sendo $10,2 \%$ do grupo pediátrico e $89,8 \%$ do grupo adulto. No grupo

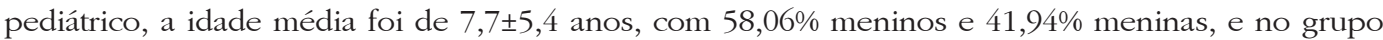
adulto a idade média foi de $61 \pm 16$ anos, com $52,38 \%$ mulheres e $47,62 \%$ homens. Perda auditiva neurossensorial foi a mais encontrada nos 2 grupos. No grupo pediátrico, perda de grau profundo foi o mais frequente e no grupo adulto, graus moderado e moderado-severo. As principais etiologias foram causas genéticas/desconhecidas no grupo pediátrico e presbiacusia no grupo adulto.

No grupo pediátrico, a maioria é de meninos, com perda neurossensorial, de graus leve e profundo, duração $\geq 1$ ano e sem comorbidades. No grupo adulto, a maioria é de mulheres, com perda neurossensorial, de graus moderado e moderado-severo, duração $\geq 5$ anos e comorbidades. audição, perda auditiva neurosensorial, prótese auditiva.

Hearing is one of the essential functions for the development of language and its deficiency may lead to several consequences.

To describe the profile of patients of the Hearing Health Program of the State of Santa Catarina attended at the College Hospital of the Federal University of Santa Catarina.

We performed a retrospective study with the patients with hearing aid indication attended in the years 2007 and 2008. They were divided into 2 groups: children ( $<18$ years) and adult ( $\geq 18$ years). We assessed the following: age, sex, loss type and degree, duration, co-morbidity and probable etiology. 304 reports were reviewed, $10.2 \%$ of the children group and $80.8 \%$ of the adult group. In the children group, the mean age was of $7.7 \pm 5,4$ years, with $58.06 \%$ boys and $41.94 \%$ girls, and in the adult group the mean age was of $61 \pm 16$ years, with $52.38 \%$ women and $47.62 \%$ men. The neurosensorial hearing loss was found more frequently in both groups. In the children group, the severe degree loss was more frequent, and in the adult group, the moderate and moderate to severe degrees were more frequent. The main etiologies were genetic/unknown causes in the children group and presbycusis in the adults group.

In the children group, most was formed by boys with neurosensorial loss of light and severe degrees, duration $\geq 1$ year and without co-morbidities. In the adult group, most was formed by women with neurosensorial loss of moderate and moderate-severe degrees, duration $\geq 5$ year and with co-morbidities. hearing, neurosensorial hearing loss, hearing impaired rehabilitation. 


\section{INTRODUÇÃO}

Conforme a Organização Mundial da Saúde (OMS), a deficiência auditiva é o mais frequente déficit sensorial na população humana, afetando mais de 250 milhões de pessoas no mundo (1). De acordo com o Instituto Brasileiro de Geografia e Estatística (IBGE), 3,37\% da população brasileira (estimada pelo censo de 2000 em 169.872 .856 pessoas) apresenta deficiência auditiva. Dados divulgados pelo IBGE de 2000 mostram que 0,8\% (406.588) dos brasileiros com idade até 14 anos apresentam perda auditiva, enquanto que $2,95 \%$ (3.240.263) dos brasileiros com idade de 15 a 64 anos e 21\% (2.088.247) dos brasileiros com 65 anos ou mais apresentam deficiência auditiva. Na população com perda auditiva e idade até 14 anos, 54,5\% são homens; já no grupo com idade entre 15 e 64 anos, a proporção é de 54,2\%; e no grupo com 65 anos ou mais, $49,75 \%$ (2).

A audição é uma das funções mais importantes para o desenvolvimento da linguagem e, portanto, para a comunicação. Entretanto, o impacto causado pela perda auditiva vem recebendo pouca atenção por parte da sociedade, governo e profissionais da saúde (3). A deficiência auditiva leva a diversas consequências, como a dificuldade para assimilar discursos, redução da capacidade para comunicação, atraso na aquisição da linguagem nas crianças, isolamento social, desvantagem educacional e econômica, e estigmatização $(1,3)$.

As perdas auditivas têm etiologias diversas e podem ser classificadas quanto ao tipo, ao grau e à idade de instalação. Quanto ao tipo, podem ser condutivas, neurossensoriais ou mistas. O grau pode ser leve, moderado, moderado-severo, severo, profundo. Quanto à idade de instalação, distinguem-se as pré-natais, as perinatais e as pós-natais $(3,4)$.

As causas pré-natais atuam durante a gestação, afetando o embrião, podendo ter como etiologias a rubéola materna, os medicamentos ototóxicos, alterações genéticas, a sífilis, a toxoplasmose e as infecções bacterianas. As causas perinatais comprometem o recém-nascido desde o início do trabalho de parto até cerca de 8 dias após o nascimento e os principais exemplos incluem o parto prolongado, a prematuridade, a anóxia e o kernicterus. As causas pós-natais são adquiridas no decorrer do desenvolvimento. Podem afetar a orelha externa, como o cerume, as infecções e os traumatismos. As alterações da orelha média podem ser: traumatismos, obstrução tubária, otites médias. Quando ocorrem prejuízos à orelha interna, a deficiência auditiva é dita neurossensorial e, dentre as principais etiologias, estão as infecções, as drogas ototóxicas e os tumores (4). Na idade adulta, ressalta-se a importância do diagnóstico de doenças como a presbiacusia e a perda auditiva induzida por ruído (5).

Devido à alta prevalência de disacusia e as consequências prejudiciais que ela pode causar, a prevenção assume um papel imprescindivel na redução desse problema (5). Além disso, a prevenção da surdez comprovadamente custa muito menos do que o seu tratamento (protetização ou implante coclear) (6). Com a finalidade de aperfeiçoar programas de prevenção e de diagnóstico de perda auditiva, o presente estudo realizou uma análise de 2 anos de atuação do Programa de Saúde Auditiva do Estado de Santa Catarina, buscando determinar o perfil do paciente atendido no Hospital Universitário da Universidade Federal de Santa Catarina, que é referência em alta complexidade deste programa, sendo responsável pela seleção e adaptação de próteses auditivas, e comparar os pacientes pediátricos com os adultos. Dessa forma, espera-se contribuir na melhoria do atendimento a este tipo de paciente.

\section{MétOdO}

O projeto deste trabalho foi aprovado pelo Comitê de Ética em Pesquisa com Seres Humanos da UFSC (projeto no 336/08).

A presente pesquisa constitui-se em um estudo transversal. Foram selecionados aleatoriamente pacientes encaminhados para atendimento no Hospital Universitário da Universidade Federal de Santa Catarina, do Programa de Saúde Auditiva do Estado de Santa Catarina, atendidos no ambulatório de Otorrinolaringologia no período de janeiro de 2007 a dezembro de 2008. Foram incluídos no estudo os pacientes com perda de audição e indicação de protetização auditiva.

Os pacientes foram classificados em dois grupos: pediátrico, com idade inferior a 18 anos, e adulto, com idade igual ou superior a 18 anos. Foram avaliados segundo a idade, sexo, tipo de perda, grau de perda, duração segundo quatro categorias (menor de 1 ano, maior ou igual a 1 ano e menor que 5 anos, maior ou igual a 5 anos e menor que 10 anos, maior ou igual a 10 anos), doenças associadas e etiologia provável. Para avaliação do tipo e grau de perda, foram coletados os dados de ambas as orelhas por paciente e avaliaram-se os exames de audiometria tonal e/ou potencial evocado auditivo do tronco encefálico (PEATE).

Na classificação do grau de perda auditiva, quando a média dos limiares tonais de 500 a $3000 \mathrm{~Hz}$ fosse normal e houvesse perda auditiva em alguma outra frequência, adotou-se o cálculo da média dos limares tonais destas frequências alteradas para classificação. Nos pacientes em 
que a avaliação da audição foi realizada apenas com PEATE, utilizou-se o limiar eletrofisiológico para classificação.

A audiometria foi realizada com os aparelhos modelos SIBELMED AC-50D, INTERACOUSTICS AC33 e AD 229e, em cabine acústica, com o uso de fone de ouvido e vibrador ósseo, avaliando-se os limiares auditivos por via aérea nas frequências de 250, 500, 1.000, 2.000, 3.000, $4.000,6.000$ e $8.000 \mathrm{~Hz}$. A via óssea foi testada nas frequências de 500, 1.000, 2.000, 3.000 e $4.000 \mathrm{~Hz}$. O potencial evocado auditivo do tronco encefálico foi realizado com o aparelho Smart Box Jr., com a utilização de estímulo sonoro tipo clique.

A análise estatística foi executada com auxílio de dois softwares: Statcalc em Epiinfo 6, utilizado para o cálculo das razões de prevalência e significância das associações calculada pelo teste do qui-quadrado no nível de confiança de $95 \%$ ( $p<0,05)$ e EpiCalc 2000, utilizado para o cálculo dos intervalos de confiança.

\section{RESULTADOS}

Foram analisados 304 prontuários de pacientes, sendo 31 (10,2\%) do grupo pediátrico e $273(89,8 \%)$ do grupo adulto. No grupo pediátrico, as idades variaram de 4 meses a 16 anos, com média de 7,7 75,4 anos. No grupo adulto, as idades variaram de 19 a 93 anos, com média de $61 \pm 16$ anos. A frequência por sexo ficou distribuída da seguinte forma: no grupo pediátrico, foram observados 18 $(58,06 \%)$ pacientes do sexo masculino e $13(41,94 \%)$ do sexo feminino, enquanto no grupo adulto foram 130 (47,62\%) pacientes do sexo masculino e $143(52,38 \%)$ do sexo feminino.

Com relação ao tipo de perda, no grupo pediátrico foram 46 perdas neurossensoriais $(74,19 \%$, IC95\%: 61,2684,10), 3 mistas (4,83\%, IC95\%: 1,26-14,38) e 1 condutiva (1,61\%, IC95\%: 0,08-9,83). Observaram-se 2 casos com audição normal (3,22\%, IC95\%: 0,56-12,17) e em 10 ocasiões os dados não estavam preenchidos (16,12\%, IC95\%: 8,41-28,13). No grupo adulto, foram 387 perdas neurossensoriais (70,87\%, IC95\%: 66,84-74,62), 145 mistas (26,55\%, IC95\%: 22,94-30,51) e 13 condutivas $(2,38 \%$, IC95\%: 1,33-4,14). Houve 1 caso com audição normal (0,18\%, IC95\%: 0,01-1,18). O resultado da análise estatística mostrou que não há diferença significativa quanto à prevalência das perdas neurossensorial e condutiva entre os dois grupos ( $\mathrm{p}=0,58 \mathrm{e} \mathrm{p}=0,70$, respectivamente). Comprovou-se estatisticamente a maior prevalência de perda mista no grupo adulto $(\mathrm{RP}=5,49 ; \mathrm{p}<0,001)$.

A distribuição dos pacientes quanto ao grau de perda é apresentada nas Figuras 1 e 2. O resultado da

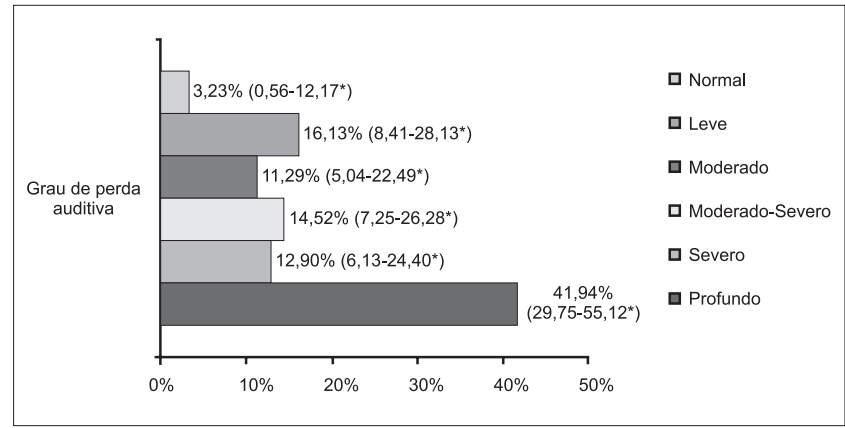

Figura 1. Grau de perda auditiva do grupo pediátrico $(n=62)$. * Intervalo de Confiança de 95\%.

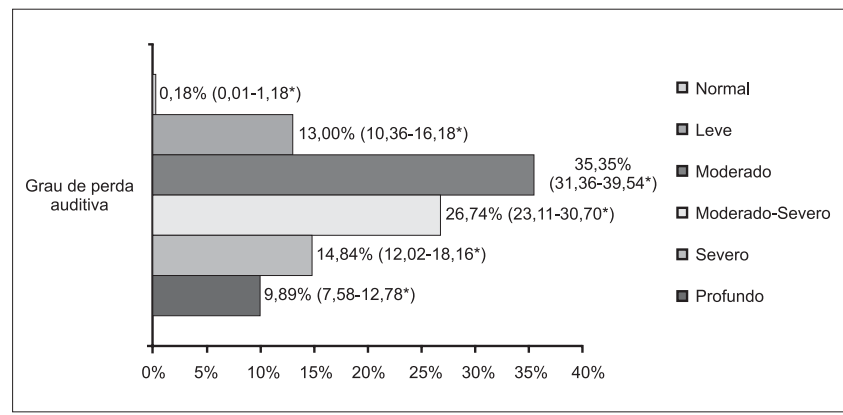

Figura 2. Grau de perda auditiva do grupo adulto $(n=546)$. * Intervalo de Confiança de 95\%.

análise estatística mostrou que não houve diferença significativa quanto à prevalência de perdas de graus leve e severo entre os dois grupos ( $\mathrm{p}=0,49$ e $\mathrm{p}=0,68$, respectivamente). Comprovou-se estatisticamente a maior prevalência de perdas de graus moderado $(\mathrm{RP}=3,13$; $\mathrm{p}<0,001)$ e moderado-severo $(\mathrm{RP}=1,84 ; \mathrm{p}=0,036)$ no grupo adulto e a maior prevalência de perda de grau profundo no grupo pediátrico $(\mathrm{RP}=4,24 ; \mathrm{p}<0,001)$.

Quanto à duração da perda, no grupo pediátrico houve $3(9,57 \%)$ casos com tempo de perda auditiva inferior a 1 ano, $10(32,25 \%)$ casos com duração igual ou maior que 1 ano e menor que 5 anos, $4(12,90 \%)$ casos com tempo de perda igual ou maior que 5 anos e menor que 10 anos, 7 (22,58\%) ocorrências de duração igual ou maior que 10 anos, sendo que, em 7 (22,58\%) pacientes, não foi possível determinar o tempo de perda. No grupo adulto, houve $1(0,36 \%)$ caso com tempo de perda auditiva inferior a 1 ano, 46 (16,84\%) casos com duração igual ou maior que 1 ano e menor que 5 anos, 39 (14,28\%) pacientes com tempo de perda igual ou maior que 5 anos e menor que 10 anos, 126 (46,15\%) ocorrências com duração igual ou maior que 10 anos, sendo que, em 61 $(22,34 \%)$ ocasiões, não foi determinado a duração da perda.

Com relação às doenças associadas, no grupo pediátrico houve $8(22,22 \%)$ casos de doenças neurológi- 
cas e mais outras 6 comorbidades (nefropatia, rinite, cardiopatia, diminuição da acuidade visual, hiperatividade e leucemia) com 1 (2,77\%) ocorrência para cada uma delas, sendo que, em 22 (61,11\%) ocasiões, os pacientes não apresentavam doenças associadas. No grupo adulto, foram 105 (30,43\%) ocorrências de hipertensão arterial sistêmica, 30 (8,69\%) de diabetes mellitus, 27 (7,82\%) de cardiopatia, 12 (3,47\%) de doenças neurológicas, 8 (2,31\%) de distúrbios psiquiátricos, $6(1,73 \%)$ de dislipidemia, 6 $(1,73 \%)$ de hipotireoidismo, $5(1,44 \%)$ de deficiência visual, $3(0,86 \%)$ de pneumopatias, $3(0,86 \%)$ de hiperplasia prostática benigna, $2(0,57 \%)$ de doenças dermatológicas, $2(0,57 \%)$ de doenças gastrointestinais, $2(0,57 \%)$ de câncer, e mais 4 outras doenças associadas (labirintopatia, osteogênese imperfeita, gota e dificuldade de locomoção) com 1 (0,28\%) ocorrência para cada, sendo que, em 130 $(37,68 \%)$ ocasiões, não foi relatada comorbidades.

A distribuição dos pacientes quanto à etiologia provável da perda auditiva é apresentada nas Figuras 3 e 4.

\section{Discussão}

Nos estudos de Nóbreg a et al (7). para o período de 1994-2000, Dereköy (8), KitTrell e Arjmand (9) demonstraram que as crianças com perda auditiva do sexo masculino predominaram sobre as do sexo feminino, respectivamente, na proporção de 1,2:1, 1,54:1 e 1,27:1. Em nosso estudo, no grupo pediátrico o sexo masculino predominou em relação ao sexo feminino na proporção de 1,38:1, em concordância com os estudos mencionados.

No estudo de Fortes et al. (5), a proporção de pacientes com hipoacusia, considerando todas as faixas etárias e os 277 pacientes atendidos (273 com hipoacusia e 4 com paralisia facial periférica), foi semelhante, com discreto predomínio para os homens (1,06:1). No estudo de AbDel-Hamid et al. (10), a proporção de homens e mulheres com perda auditiva com idade igual ou superior a 15 anos foi semelhante, com leve predomínio das mulheres (1,08:1). Em nosso levantamento, a proporção de mulheres no grupo adulto foi levemente maior do que a dos homens $(1,1: 1)$.

Cесатто et al. (6) constataram, através da análise de 131 prontuários de alunos frequentadores de uma escola para surdos, que a perda auditiva neurossensorial foi a mais comum, representando 99\% dos casos. LeE et al. (11) avaliaram 234 pacientes que pleitearam próteses auditivas e, das 468 orelhas analisadas, $77,56 \%$ apresentavam perda auditiva neurossensorial, sendo esse tipo de perda a mais encontrada em todas as faixas etárias. Também em nossa amostra a deficiência auditiva neurossensorial foi a mais

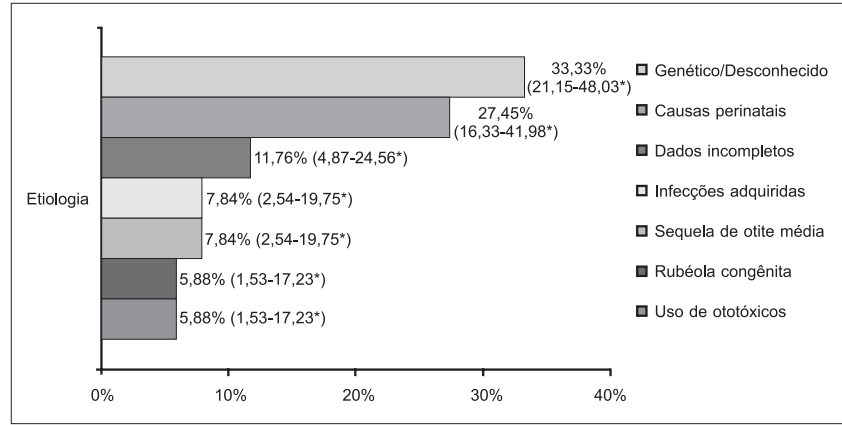

Figura 3. Etiologia provável no grupo pediátrico $(n=51)$.

* Intervalo de Confiança de 95\%.

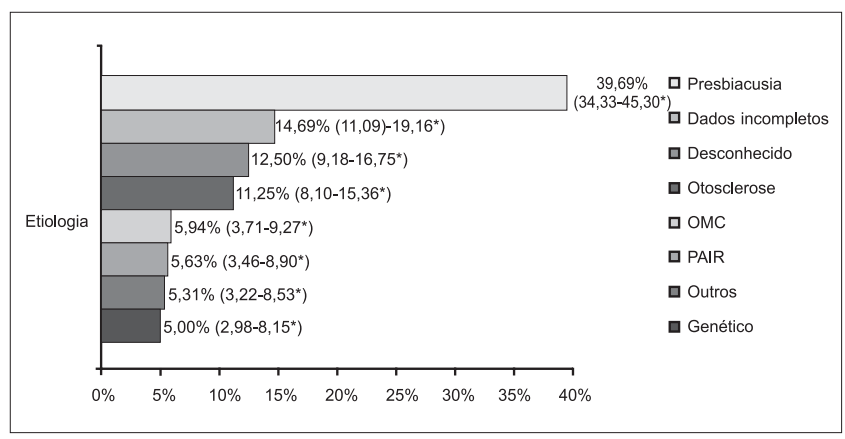

Figura 4. Etiologia provável no grupo adulto $(n=320)$. * Intervalo de Confiança de 95\%.

comum, representando $74,19 \%$ e $70,87 \%$ nos grupos pediátrico e adulto, respectivamente.

No levantamento de KitTrell e Arjmand (9), que avaliaram 291 deficientes auditivos com idades variando de 4 a 20 anos, a perda auditiva de grau profundo representou $82 \%$ dos casos. No estudo de CECATTO et al. (6), a deficiência de grau profundo ocorreu em 65\%. Em nosso trabalho, comprovamos estatisticamente a maior prevalência de perda de grau profundo no grupo pediátrico.

No estudo de Veras e Mattos (12), 69,6\% dos idosos com perda auditiva apresentaram perda de grau leve considerando a melhor orelha. Wilson et al. (13) estimaram a prevalência de deficiência auditiva em pessoas com 15 anos ou mais e observaram, considerando a melhor orelha, que a perda de grau leve também foi a mais frequente. Em nossa pesquisa, diferentemente dos estudos citados, os graus moderado e moderado-severo prevaleceram no grupo adulto, provavelmente pelo fato de os pacientes terem indicação do uso de prótese auditiva, o que significa maior gravidade da deficiência apresentada.

O diagnóstico precoce das deficiências auditivas na criança exige uma anamnese detalhada (4). Assim como no protocolo utilizado no presente estudo, deveria ser inclu- 
ído na história questões sobre infecções, uso de drogas ototóxicas, dados a respeito do nascimento (prematuridade, anóxia, hiperbilirrubinemia), pesquisa de deformidades gerais e de casos de deficiência auditiva na família (4).

Em muitas situações, mesmo com uma investigação minuciosa, a etiologia precisa da perda auditiva não pode ser definida. Nos estudos de CeCatto et al. (6), DeReköy (8) e WALCH et al. (14) não apresentaram etiologia definida respectivamente $33(25,2 \%)$ pacientes, $34(26,1 \%)$ crianças e $47(44 \%)$ crianças.

A alta incidência de etiologia desconhecida no grupo pediátrico nesse levantamento corrobora com os estudos descritos e demonstra que a aplicação de questionário padronizado falhou na determinação da etiologia. A confirmação do diagnóstico etiológico de perda auditiva ainda constitui um grande desafio para a maioria dos pesquisadores (7). Em nosso trabalho, essas dificuldades na identificação da etiologia também foram encontradas no grupo adulto, já que as causas desconhecidas representaram a segunda causa mais comum de deficiência auditiva nesse grupo.

As causas genéticas de perda auditiva estão presentes na maior parte dos estudos (7). No estudo de NóBreGa et al. (7), a perda genética ocorreu em $15,73 \%$ dos pacientes no período de 1990-1994, e em 8,33\% das crianças nos anos de 1994-2000. No estudo de DereKöy (8), realizado em uma escola para surdos, a etiologia genética representou a terceira causa mais comum de deficiência auditiva, com 31 (23,8\%) crianças afetadas, atrás apenas de convulsão febril com 35(26,9\%) e causa desconhecida com $34(26,1 \%)$ crianças. Constatou-se no presente estudo que a etiologia genética/desconhecida, representada em 33,33\% dos casos no grupo pediátrico, foi a causa mais expressiva de deficiência auditiva, confirmando a importância de ambas etiologias nesse grupo.

As principais causas perinatais responsabilizadas pela deficiência auditiva incluem a anóxia, a prematuridade, a eritroblastose fetal e o kernicterus (4). De acordo com STREPPEl et al. (15), o acúmulo de causas perinatais é a razão da maioria das etiologias adquiridas de deficiência auditiva nas crianças alemãs. Segundo esses autores, o avanço da neonatologia trouxe a diminuição da mortalidade perinatal e o aumento das complicações perinatais, como a perda auditiva adquirida.

Em nosso estudo, as causas perinatais representaram a segunda causa mais comum de surdez no grupo pediátrico e esse progresso da pediatria é uma possível explicação para esses achados. A análise estatística mostrou que não há diferença significativa entre a frequência das causas genéticas/desconhecidas e as perinatais no grupo pediátrico. Já no grupo adulto, as causas genéticas e perinatais foram infrequentes, demonstrando a importância de outras etiologias nessa faixa etária, como a presbiacusia, a PAIR, a otosclerose e a OMC.

A presbiacusia, perda auditiva devido à idade, é apontada como a principal causa de deficiência auditiva em idosos na literatura internacional, com uma incidência de cerca de 30\% na população com mais de 65 anos de idade (16). De acordo com Veras e Mattos (12), que realizaram uma revisão da literatura sobre esse tema, a presbiacusia vem sendo considerada a causa mais comum de deficiência auditiva em pessoas idosas no Brasil, implicando numa dificuldade de compreensão durante a comunicação verbal. Em nosso trabalho, a presbiacusia foi a principal causa de perda auditiva no grupo adulto, mostrando concordância com as referências citadas. Comprovou-se estatisticamente que a presbiacusia é a etiologia mais frequente no grupo adulto.

A perda auditiva induzida pelo ruído (PAIR) é a principal causa de surdez e perda auditiva nos Estados Unidos. Embora o envelhecimento e a genética sejam os principais fatores de risco, a perda auditiva temporária ou permanente está se tornando cada vez mais comum entre adultos jovens e crianças, sobretudo com o aumento da exposição a aparelhos de música portáteis (17). Dessa forma, devem ser educados e orientados a evitar o uso desses equipamentos. Em nosso estudo, não foi encontrado nenhum caso de PAIR no grupo pediátrico, enquanto que no grupo adulto a PAIR foi responsável por 5,63\% das causas de perda auditiva.

Otosclerose é uma das mais frequentes causas de perda auditiva no indivíduo adulto (18). É mais frequente no sexo feminino, na proporção de $2: 1$, podendo apresentar piora dos sintomas durante a gravidez $(18,19)$. Sua incidência é mais comum entre 20 e 40 anos, sendo infrequente em crianças e adultos com mais de 50 anos $(18,20)$. Em nossa pesquisa, a incidência de otosclerose foi mais frequente nas mulheres, na proporção de 2:1, com $41,6 \%$ dos pacientes na faixa etária dos 20 aos 50 anos, sendo ausente antes dos 35 anos. É possível que a maior parte dos casos após os 50 anos seja em parte justificada pelas indicações de protetização auditiva na otospongiose e pelo tempo de espera para marcação da consulta, já que o HU é referência estadual do Programa de Saúde Auditiva. Uma das principais indicações para o uso de próteses auditivas na otosclerose é naqueles pacientes que não podem submeter-se ao tratamento cirúrgico, sendo que a maioria desses são idosos. Também nos idosos, é mais frequente a otosclerose associada ao acometimento neurossensorial, onde a cirurgia não modifica tal perda, sendo a indicação de prótese auditiva mais comum. 
No estudo de Fortes et al. (5), a etiologia infecciosa representou aproximadamente $70 \%$ dos casos de deficiência auditiva na faixa etária inferior a 40 anos, diminuindo com o avanço da idade, mas ainda representando $31 \%$ dos casos na população acima de 60 anos, sendo a grande maioria dos casos de otite média crônica (79\%) e secretora (18\%) e apenas $1,2 \%$ dos casos otite média aguda. AbdelHamid et al. (10), em uma pesquisa com 4000 egípcios para estimar a prevalência e as causas de perda auditiva no Egito, constataram que $16,02 \%$ (641) dos participantes apresentavam perda auditiva e as 3 causas mais comuns foram: otite média com efusão $(30,7 \%)$, presbiacusia $(22,7 \%)$ e otite média crônica supurativa (13,2\%).

Em nossa pesquisa, a OMC, que foi incluída no subgrupo sequela de otite média no grupo pediátrico, ocorreu em 3 pacientes desse grupo e em 19 (5,94\%) pacientes do grupo adulto, representando a terceira causa identificável mais frequente de perda auditiva nesse último grupo. É possível que esses casos de OMC em nosso trabalho, proporcionalmente menor do que nos estudos citados, tenha ocorrido por várias razões, entre elas o fato de analisarmos apenas pacientes com indicação de protetização auditiva. Isso permite que as etiologias cujo tratamento fundamenta-se principalmente no uso de aparelhos de amplificação sonora individual, como a presbiacusia, sobressaíam-se em relação a causas em que o tratamento clínico e cirúrgico sejam eficazes em grande número de pacientes, como nos casos de otite média crônica, incluindo a otite média com efusão.

Infecções adquiridas são outras importantes causas de perda auditiva. Entre as infecções adquiridas, a meningiteé, sem dúvida, a principal e apontada como a etiologia pós-natal mais relevante de disacusia neurossensorial $(6,15)$. Nos estudos de CECATto (6) e Butugan (21), foram avaliados pacientes com idades variando de 3 a 30 anos e no primeiro ano de vida respectivamente, e a incidência de deficiência auditiva por meningite foi de $8,4 \%$ em ambos trabalhos. Foram atendidos nesse estudo 3 casos de meningite no grupo pediátrico, representando, juntamente com a otite média crônica, a causa pós-natal mais comum de déficit auditivo nessa faixa etária.

Segundo Nóbrega et al. (7), a rubéola congênita ainda é uma das principais etiologias de perda auditiva em nosso meio. CесатTо et al. (6) descreveram que a rubéola congênita é a principal causa pré-natal de perda auditiva pelos seus efeitos teratogênicos e demonstrou que a rubéola congênita correspondeu a 31 (23,6\%) dos casos e foi a causa identificável mais comum de deficiência auditiva. NóbREg a et al. (7) constataram, durante o período de 1990-1994, que a rubéola congênita foi a segunda causa mais comum de perda auditiva em crianças e adolescentes, sendo precedida apenas pela etiologia desconhecida.
No estudo de Dereköy (8), que avaliou 130 estudantes surdos com idades variando de 5 a 16 anos em uma escola na Turquia, não houve nenhum caso de rubéola materna como causa de surdez. Similarmente, não houve síndrome da rubéola congênita em outros 6 estudos na Turquia. Como nesse país ainda não foi adotado um programa de imunização contra a rubéola, a imunidade natural contra a rubéola é alta. De acordo com STREPPEL $\boldsymbol{e t}$ al. (15), a redução dos casos de rubéola congênita detectados na Alemanha parece ser devido ao sucesso da vacinação.

Em nosso estudo, a rubéola congênita representou, no grupo pediátrico, a causa menos comum de deficiência auditiva juntamente com o uso de ototóxicos, mostrando a discordância com estudos nacionais, mas de acordo com os estudos turco e alemão. Nos estudos realizados por NóBREGA (7) e CéatTo (6), os pacientes foram avaliados nos anos de 1990-2000 e no ano de 2001, respectivamente. As ações de vacinação de 2001-2002 do Ministério da Saúde do Brasil, sendo direcionadas, de maneira geral, às mulheres de 12 a 39 anos levaram a redução da incidência da rubéola. Entretanto, essa cobertura vacinal não foi homogênea, acumulando suscetíveis e contribuindo para os surtos de 2006 e 2007 (22).

Esses surtos não atingiram grandes repercussões em Santa Catarina, que apresentou 92 casos confirmados de rubéola nos anos de 2006 e 2007, em comparação com outros estados, a exemplo do Rio Grande do Sul, que teve 2866 casos nesse mesmo período $(23,24)$. Com o objetivo de interromper a circulação do vírus da rubéola no Brasil, o Ministério da Saúde promoveu a campanha vacinal de 2008 para homens e mulheres, direcionada a faixa etária de 20-39 anos, que ficou definida como população suscetível (22). A tendência é que em anos subsequentes haja uma queda significativa dos casos de rubéola no país e, consequentemente, de síndrome da rubéola congênita.

Em relação à ototoxicidade na infância, são os recém-nascidos com infecções graves no berçário de terapia intensiva que recebem antibióticos aminoglicosídeos. Vários autores têm publicado porcentagens variáveis de casos de ototoxicoses em crianças, decorrentes do uso de vários antibióticos aminoglicosídeos. Outros autores relatam ausência de ototoxicose em crianças que receberam essas drogas (3). Em nosso levantamento, foram atendidos $3(5,88 \%)$ casos de perda auditiva no grupo pediátrico, tendo como etiologia provável o uso de ototóxicos, sendo que em 2 crianças empregou-se a gentamicina.

\section{CONCLUSÃO}

A maioria dos pacientes do grupo pediátrico enca- 
minhados para atendimento no Hospital Universitário da UFSC, do Programa de Saúde Auditiva do Estado de Santa Catarina, são do sexo masculino, com idade média de 7,7 anos, com perda auditiva neurossensorial, de graus leve e profundo, com duração da perda igual ou maior do que 1 ano e sem doenças associadas. As etiologias genéticas/ desconhecidas e as causas perinatais representaram mais de $60 \%$ de todas as causas desse grupo. A maior parte dos pacientes do grupo adulto encaminhados para atendimento são do sexo feminino, com idade média de 61 anos, com perda auditiva neurossensorial, de graus moderado e moderado-severo, com duração da perda igual ou maior do que 5 anos, sendo a hipertensão arterial sistêmica a doença associada mais frequente. A presbiacusia foi a etiologia mais comum no grupo adulto.

Comparando os dois grupos houve maior prevalência da perda auditiva do tipo misto no grupo adulto. Quanto ao grau, ocorreu maior prevalência de perdas de graus moderado e moderado-severo no grupo adulto e profundo, no grupo pediátrico. As principais etiologias identificáveis foram distintas nos 2 grupos.

\section{REFERÊNCIAS BIBLIOGRÁFICAS}

1. World Health Organization [homepage na Internet]. Mathers C, Smith A, Concha M. Global Burden of hearing loss in the year 2000 [acesso em 2009 Out 29]. Disponível em: www.who.int/entity/healthinfo/statistics/ bod_hearingloss.pdf

2. Instituto Brasileiro de Geografia e Estatística [homepage na Internet]. Censo de 2000 [acesso em 2009 Out 29]. Disponível em: http://www.ibge.gov.br/home/estatistica/ populacao/ censo $2000 /$ populaca o/ censo2000_populacao.pdf

3. Costa SS, Cruz OLM, Oliveira JAA. Otorrinolaringologia: Princípios e Prática. $2^{\mathrm{a}}$ ed. Porto Alegre: Artmed, 2006.

4. Costa SS, Cruz OLM, Oliveira JAA. Otorrinolaringologia: Princípios e Prática. $1^{\mathfrak{a}}$ ed. Porto Alegre: Artes Médicas, 1994.

5. Fortes FSG, Franceso RC, Bento RF, Miniti A. Liga de Prevenção à Surdez: Análise de Três Anos de Atuação. Arq Int Otorrinolaringol. 2002 , 6(4):302-9.

6. Cecatto SB, Garcia RID, Costa KS, Abdo TRT, Rezende CEB, Rapoport PB. Análise das principais etiologias de deficiência auditiva em Escola Especial "AnneSullivan”. Rev Bras Otorrinolaringol. 2003, 69(2):235-40.

7. Nóbrega M, Weckx LLM, Juliano Y. Study of the hearing loss in children and adolescents, comparing the periods of
1990-1994 and 1994-2000. Int J Pediatr Otorhinolaryngol. 2005, 69(6):829-38.

8. Dereköy FS. Etiology of deafness in Afyon school for the deaf in Turkey. Int J Pediatr Otorhinolaryngol. 2000, 55(2):125-31.

9. Kittrell AP, Arjmand EM. The age of diagnosis of sensorineural hearing impairment in children. Int J Pediatr Otorhinolaryngol. 1997, 40(2-3):97-106.

10. Abdel-Hamid O, Khatib OMN, Aly A, Morad M, Kamel S. Prevalence and patterns of hearing impairment in Egypt: a national household survey. East Mediterr Health J. 2007, 13(5):1170-80.

11. Lee IWCC, Brasileiro HMS, Boldorini PR, Rapoport A, Novo NF. Perfil epidemiológico das deficiências auditivas no interior de São Paulo. Estudo de 234 casos. Rev Bras Cir Cabeça Pescoço. 2004, 33(2):89-92.

12. Veras RP, Mattos LC. Audiologia do envelhecimento: revisão da literatura e perspectivas atuais. Rev Bras Otorrinolaringol. 2007, 73(1):122-8.

13. Wilson DH, Walsh PG, Sanchez L, Davis AC, Taylor AW, Tucker $\mathrm{G}$ et al. The epidemiology of hearing impairment in an Australian adult population. Int J Epidemiol. 1999, 28(2):247-52.

14. Walch C, Anderhuber W, Köle W, Berghold A. Bilateral sensorineural hearing disorders in children: etiology of deafness and evaluation of hearing tests. Int J Pediatr Otorhinolaryngol. 2000, 53(1):31-8.

15. Streppel M, Richling F, Roth B, Walger M, von Wedel H, Eckel HE. Epidemiology and etiology of acquired hearing disorders in childhood in the Cologne area. Int J Pediatr Otorhinolaryngol. 1998, 44(3):235-43.

16. Política Nacional de Saúde da Pessoa Portadora de Deficiência [homepage na Internet]. Portaria n. 1.060, de 5 de junho de 2002. Diário Oficial, Brasília (2002 jun 10).[acesso em 2009 Out 29]. Disponível em: http:// portal.saude.gov.br/portal/arquivos/pdf/manual2.pdf

17. Daniel E. Noise and Hearing Loss: A Review. JSch Health. 2007, 77(5):225-31.

18. Fukuda Y. Otorrinolaringologia. $1^{a}$ ed. São Paulo: Manole, 2003.

19. Jafek BW, Murrow BW. Segredosem Otorrinolaringologia: respostas necessárias ao dia-a-dia em rounds, na clínica, em exames orais e escritos. $2^{\mathrm{a}}$ ed. Porto Alegre: Artmed, 2006. 
20. Hungria H. Otorrinolaringologia. $7^{a}$ ed. Rio de Janeiro: Guanabara Koogan,1995.

21. Butugan O, Santoro PP, Almeida ER, Silveira JAM, Grasel SS. Diagnóstico precoce da deficiência auditiva no primeiro ano de vida de crianças comalto risco através de audiometria de tronco cerebral. Pediatria (São Paulo). 2000, 22(2):11522.

22. Brasil livre da rubéola: campanha nacional de vacinação para eliminação da rubéola, Brasil, 2008: relatório/Ministério da Saúde, Secretaria de Vigilância em Saúde, Departamento de Vigilância Epidemiológica.-Brasília: Ministério da Saúde, 2009.
23. Secretaria de Vigilância em Saúde do Ministério de Saúde do Brasil [homepage na Internet]. Casos confirmados de Rubéola, Brasil, Grandes Regiões e Unidades Federadas 1997 a 2006 [acesso em 2009 Out 29]. Disponível em: http:// portal.saude.gov.br/portal/arquivos/pdf/casos_rubeola.pdf.

24. Secretaria de Vigilância em Saúde do Ministério de Saúde do Brasil [homepage na Internet]. Casos confirmados de Rubéola, segundo mês de início de sintomas, Brasil, Grandes Regiões e Unidades Federadas 2007 [acesso em 2009 Out 29]. Disponível em: http://portal.saude.gov.br/portal/ arquivos/pdf/casos_conf_rubeola_mes_sintomas2007.pdf. 